\title{
L'intégration de l'EMDR dans une psychothérapie évolutionniste novatrice : une étude de cas de dépression post-partum
}

\author{
Valery Krupnik \\ Hôpital Naval Camp Pendleton, Californie, États-Unis
}

\begin{abstract}
La dépression constitue l'un des troubles psychiatriques les plus fréquents. La dépression post-partum affecte environ $9 \%$ des femmes qui accouchent. Malgré des progrès importants en recherche, pharmacothérapie et psychothérapie, les troubles dépressifs demeurent difficiles à traiter. L'application de la psychothérapie EMDR (désensibilisation et retraitement par les mouvements oculaires) à la dépression connaît un retard par rapport à son utilisation dans le traitement de troubles anxieux ou dans le domaine du trauma. Je présente deux cas de dépression post-partum qui ont été traités avec succès à l'aide d'une psychothérapie combinée intégrant l'EMDR dans un cadre psychothérapeutique novateur, développé spécialement pour les troubles dépressifs et fondé sur la théorie évolutionnaire de la dépression : « traiter la dépression en descente " (treating depression downhill [TDD]). Dans la psychothérapie intégrée TDD-EMDR, j'ai apporté quelques ajustements au protocole EMDR standard, de telle sorte que le choix et le type de cibles, le cadre cognitif et le changement affectif attendu sont déterminés par le cadre TDD. Les cas décrits illustrent le processus thérapeutique, y compris les modifications apportées aux procédures EMDR standard ainsi que les résultats du traitement. J'identifie et traite des différences entre la théorie de l'EMDR et celle du TDD.
\end{abstract}

Mots-clés : EMDR ; traiter la dépression en descente (TDD) ; dépression post-partum ; psychothérapie intégrative ; évolution

ans le DSM-5 (Diagnostic and Statistical Manual of Mental Disorders, $5^{\mathrm{e}}$ édition), la dépression post-partum est incluse dans le trouble dépressif majeur avec apparition péri-partum (American Psychiatric Association [APA], 2013). De 8,5\% à $11 \%$ des femmes souffrent d'une dépression majeure pendant la grossesse et de 6,5 à $12 \%$ de dépression post-partum (Gaynes et coll., 2005). En plus des facteurs environnementaux généraux qui contribuent à son étiologie, la dépression post-partum en possède aussi plusieurs qui sont propres à la maternité. Ils comprennent la grossesse non prévue et non désirée, le manque de soutien de la part d'un partenaire, la qualité de la relation avec ce partenaire et le stress de s'occuper d'un enfant (C. T. Beck, 2001 ; Cortés-Salim, González-Barrón \& Romero-Gutiérrez, 2014). Le bien-être des enfants de la mère affectée constitue une préoccupation importante car on pense que la dépression post-partum comporte des conséquences négatives à long terme pour les enfants (Murray et coll., 2011).

La grossesse et la période post-partum constituent des moments de grande vulnérabilité et d'enjeux évolutionnaires importants pour la femme et pour sa progéniture. Ce fait a conduit à la notion de la signification de la dépression post-partum en tant que mécanisme évolutionnaire visant à réduire

This article originally appeared as Krupnik, V. (2015). Integrating EMDR Into a Novel Evolutionary-Based Therapy for Depression: A Case Study of Postpartum Depression. Journal of EMDR Practice and Research, 9(3), 137-149. Translated by Jenny Ann Rydberg. 
ou à éliminer l'investissement de la femme envers ses petits lors de conditions défavorables, par le biais d'un détachement ou d'une agressivité vis-à-vis des enfants (Hagen, 1999). Selon les résultats d'une étude à ce sujet, l'ocytocine, une hormone impliquée dans l'attachement mère-enfant (Feldman, Weller, Zagoory-Sharon \& Levine, 2007) joue un rôle dans la dépression post-partum, des niveaux faibles pendant la grossesse étant corrélés avec le risque de dépression post-partum (Skrundz, Bolten, Nast, Hellhammer \& Meinlschmidt, 2011). Ce résultat indique le rôle intermédiaire que pourrait jouer une insuffisance en ocytocine dans le détachement maternel à l'égard de ses enfants lorsque soumise à un stress important.

\section{Une approche évolutionniste dans le traitement de la dépression}

Le trouble dépressif majeur demeure l'un des troubles mentaux les plus répandus mais aussi l'un des plus difficiles à traiter. Plus de $20 \%$ des cas ne répondent pas aux traitements conventionnels (Fava, 2003 ; Rush et coll., 2006) et ce malgré l'abondance de thérapies destinées au traitement de la dépression qui se sont récemment développées en termes de diversité et de sophistication (Jorm, Allen, Morgan \& Purcell, 2009). Cependant, parmi les nombreux traitements employés, aucun ne semble vraiment sortir du lot au niveau de leur succès (Braun, Gregor \& Tran, 2013; Shedler, 2010).

En se basant sur les théories évolutionnaires de la dépression (P. W. Andrews \& Thompson, 2009 ; Gilbert, 2006 ; Keller \& Nesse, 2006 ; Nettle, 2004 ; Watt \& Panksepp, 2009), Krupnik (2014) a affirmé que le désengagement hédonique, motivationnel et comportemental dans la dépression remplit une fonction adaptative et peut donc contribuer à la résistance au traitement de la dépression rebelle. Le traitement d'un individu fortement replié sur lui-même, présentant principalement des symptômes négatifs, ce qui est caractéristique d'une dépression (DSM-5), peut en effet présenter des difficultés.

Il se peut également qu'en vue d'une guérison, la réponse dépressive doit d'abord suivre son cours pour permettre à dépression de "toucher le fond ". Ainsi, Watt et Panksepp (2009) ont émis l'hypothèse que la transition depuis la protestation jusqu'au désengagement constitue la fonction de la réponse dépressive. Ce point de vue sous-tend le développement d'un nouveau cadre psychothérapeutique (Krupnik, 2014): traiter la dépression en descente (TDD).

Une autre psychothérapie de la dépression évolutionniste, la thérapie cognitive fondée sur l'évolution (evolutionary-driven cognitive therapy; TC-FE), a été mise au point depuis peu. La TC-FE représente une modification de la thérapie cognitive comportementale (TCC) qui cible ce qui met en péril le bien-être du patient au moyen de la restructuration cognitive et de la modification comportementale (Giosan et coll., 2014).

\section{Traiter la dépression en descente}

La psychothérapie TDD se fonde sur la théorie évolutionnaire de la dépression qui considère la réponse dépressive comme une adaptation à des épreuves insurmontables. Les preuves préliminaires montrent l'efficacité de la TDD dans le traitement de la dépression ainsi que sa spécificité, la TDD n'étant pas efficace pour l'anxiété ou les troubles de la personnalité (Krupnik, 2014). Elle consiste en trois phases : exploration, acceptation et activation comportementale.

Dans la phase exploratoire, les patients développement une compréhension des origines de leurs réactions dépressives. Ils identifient les principaux thèmes dépressogènes qui dominent leur récit, révélant leur connexion aux concepts d'échec et de perte. Dans la deuxième phase, l'acceptation, les patients se défont de la protestation en développant un état d'esprit porté à l'acceptation. Cette phase vise à favoriser la réponse dépressive pour écourter la période où elle touche à son point le plus bas (Krupnik, 2014). Elle repose sur des techniques de pleine conscience utilisées dans plusieurs thérapies pour cibler les symptômes de la dépression et de l'anxiété (résumées dans S. G. Hofmann, Sawyer, Witt \& Oh, 2010). Dans la TDD, la pleine conscience est appliquée pour aider les patients à se défaire de l'état d'esprit qui proteste et pour favoriser l'acceptation de l'échec et de la perte face à des épreuves insurmontables qui leur sont arrivées tant dans le présent que dans le passé. La perte et l'échec sont des concepts psychologiques fondamentaux censés sous-tendre la dépression (Bowlby, 1980 ; Gilbert \& Allan, 1998 ; Watt \& Panksepp, 2009).

Lors de la troisième phase, celle de l'activation, les patients utilisent des stratégies comportementales pour devenirs plus motivés, actifs et engagés. La thérapie d'activation comportementale a déjà montré son efficacité dans le traitement de la dépression (Jacobson, Martell \& Dimidjian, 2001). En tant que psychothérapie intégrative, la TDD combine les techniques des thérapies psychodynamiques, de pleine conscience, comportementales et cognitives (Krupnik, 2014). Il est intéressant de noter que la transition depuis la protestation jusqu'à l'acceptation correspond plus ou moins aux étapes du processus de deuil (Kübler-Ross, 1997). 


\section{L'EMDR dans le traitement des troubles dépressifs}

L'EMDR (désensibilisation et retraitement par les mouvements oculaires) est une psychothérapie intégrative comportant un volet expérientiel important, développé à l'origine pour le traitement du stress traumatique (F. Shapiro, 1989). Elle a démontré son efficacité et est reconnue comme l'un de traitements fondés sur les preuves de l'état de stress post-traumatique (ESPT ; p. ex. Bisson, Roberts, Andrew, Cooper \& Lewis, 2013).

La thérapie EMDR est une approche psychothérapeutique en huit phases, comportant des protocoles et des procédures standardisés. Les huit phases et le protocole en trois volets favorisent une évaluation complète du tableau clinique, la préparation du patient et le traitement a) d'événements passés qui ont jeté les bases de la pathologie, b) de situations perturbantes actuelles et c) de défis futurs. (F. Shapiro, 2014, p. 72)

Le traitement adaptatif de l'information (TAI) est la théorie qui sous-tend l'EMDR (F. Shapiro, 2001 ; Solomon \& Shapiro, 2008). La théorie considère que des souvenirs stockés de manière dysfonctionnelle sont à l'origine de la psychopathologie parce qu'ils interfèrent avec le fonctionnement présent. C'est pourquoi on pense que le retraitement de ces souvenirs à l'aide de l'EMDR aide à les transformer et à les intégrer dans des réseaux neuraux plus vastes, les rendant ainsi plus adaptatifs et moins perturbateurs. La stimulation sensorielle bilatérale (SBL) est considérée comme favorisant un tel retraitement.

Depuis sa création, les applications de l'EMDR se sont multipliées bien au-delà des troubles traumatiques pour inclure, entre autres, des troubles affectifs, de la personnalité et des conduites alimentaires (R. E. Shapiro, 2009).

L'application de l'EMDR aux troubles dépressifs a le plus souvent été rapportée dans des études de cas cliniques utilisant le protocole standard et démontrant son efficacité dans le soulagement des symptômes dépressifs (Bae, Kim \& Park, 2008 ; Broad \& Wheeler, 2006 ; Grey, 2011 ; Uribe, Ramírez \& Mena, 2010). En particulier, dans le cas de deux adolescents traités en EMDR, les symptômes se sont résorbés assez rapidement : après trois ou sept séances (Bae et coll., 2008).

Dans un essai contrôlé randomisé, l'ajout de la psychothérapie standard augmentait l'efficacité de la TCC dans le traitement de la dépression (A. Hofmann et coll., 2014). Pourtant, l'application de l'EMDR à la dépression présente du retard par rapport à son utilisation pour les troubles anxieux et liés au trauma.

\section{Intégration des psychothérapies TDD et EMDR}

Les psychothérapies intégratives sont devenues le pilier de la pratique contemporaine. Il existe différentes stratégies de l'intégration de psychothérapies (Castonguay, Reid, Halperin \& Goldfried, 2003). L'intégration par assimilation fondée sur des principes (Castonguay et coll., 2003 ; Messer, 2001) repose sur des principes théoriques qui traversent différentes orientations méthodologiques pour combiner certains éléments de leurs techniques en une psychothérapie cohérente. Ici, je présente une tentative d'intégration de la TDD et de l'EMDR, dont chacune est déjà intégrative. Les principes unificateurs de mon approche de cette intégration sont (a) l'identification et le ciblage d'expériences propres à la pathologie du patient, c'est-à-dire des moments de perte et d'échec et (b) des interventions expérientielles pour mettre en œuvre le changement thérapeutique. La justification principale du développement de la TDD était la conception d'un traitement spécifique à la dépression en ciblant les processus psychologiques qui animent la réponse dépressive (Krupnik, 2014).

L'EMDR est une psychothérapie centrée sur le trauma, dont il a été prouvé qu'elle produit des gains plus durables, lorsqu'appliquée à l'ESPT, que des traitements moins spécifiques et non centrés sur le trauma

(Bisson et coll., 2013). Ainsi, tant l'EMDR que la TDD visent une approche spécifique à la pathologie. La méditation de pleine conscience est pratiquée dans la phase d'acceptation de la TDD, tandis que l'EMDR recourt à la pleine conscience pendant les SBL (F. Shapiro, 2001). Dans notre expérience, tout le monde n'est ni prêt ni capable à pratiquer la méditation de pleine conscience dans son état dépressif. Williams, Teasdale, Segal et Kabat-Zinn (2007) mettent également en garde : « . . il ne serait pas conseillé d'entreprendre tout le programme [pratique de la pleine conscience] en se trouvant en plein milieu d'un épisode de dépression clinique " (p. 8). C'est pourquoi, dans la psychothérapie combinée TDD-EMDR, j'ai remplacé les interventions EMDR, qui incorporent la pleine conscience par les SBL, par la méditation en pleine conscience dans la phase d'acceptation. Une autre caractéristique commune à la TDD et à l'EMDR correspond à des éléments d'exposition, qui se retrouve dans plusieurs psychothérapies, y compris celles qui ne sont pas considérées comme des thérapies d'exposition. En reconnaissance de cette tendance, Harper (2012) a proposé le terme de thérapie de dépotentialisation 
pour désigner toutes les psychothérapies utilisant l'exposition. Une technique d'exposition vient d'être intégrée à la thérapie cognitive pour développer une nouvelle thérapie de la dépression, la thérapie cognitive fondée sur l'exposition (Holtforth et coll., 2012).

\section{L'application de la TDD-EMDR à la dépression post-partum}

L'application de la TDD-EMDR à un cas de dépression majeur a déjà été décrite ailleurs (Krupnik, 2015) ; ce texte présente une application de la TDD-EMDR à la dépression post-partum. À ma connaissance, il s'agit de la première description d'une application de l'EMDR à la dépression post-partum. La TDD-EMDR suit le schéma de la TDD (Krupnik, 2014). Pour assimiler l'EMDR dans le cadre TDD, des modifications ont été apportées au protocole EMDR standard. Je conçois la pathologie dépressive comme une réponse dépressive interrompue ou incomplète, déclenchée lorsque la personne échoue à satisfaire ses besoins. Cet insuccès se manifeste psychologiquement comme un sentiment de perte et d'échec. Par conséquent, le but de la psychothérapie est de mener jusqu'à son terme la réponse dépressive en se désengageant d'abord de la protestation, grâce à l'acceptation, puis en restaurant la motivation par l'activation comportementale (tableau 1). La TDD-EMDR consiste en les mêmes trois phases que la TDD : exploration, acceptation et activation comportementale.

TABLEAU 1. Comparaison des fondements théoriques de l'EMDR et de la thérapie Traiter la dépression en descente (TDD)

\begin{tabular}{ccc}
\hline & EMDR (TAI) & TDD \\
\hline $\begin{array}{c}\text { Origine de la } \\
\text { pathologie }\end{array}$ & $\begin{array}{c}\text { Souvenirs stockés } \\
\text { de manière } \\
\text { dysfonctionnelle }\end{array}$ & $\begin{array}{c}\text { Besoins } \\
\text { inassouvis }\end{array}$ \\
$\begin{array}{ccc}\text { But de la } \\
\text { thérapie }\end{array}$ & $\begin{array}{c}\text { Retraitement adap- } \\
\text { tatif de souvenirs } \\
\text { dysfonctionnels }\end{array}$ & $\begin{array}{c}\text { Achèvement de } \\
\text { la réponse } \\
\text { dépressive }\end{array}$ \\
$\begin{array}{ccc}\text { Le mécanisme } \\
\text { de change- } \\
\text { ment supposé }\end{array}$ & $\begin{array}{c}\text { Transformation souvenirs } \\
\text { dysfonctionnels } \\
\text { et leur intégra- }\end{array}$ & $\begin{array}{c}\text { Désengagement } \\
\text { de la protes- } \\
\text { tion dans des }\end{array}$ \\
& l'acceptation par \\
& réseaux adapta- & \\
& tifs plus vastes & \\
\hline
\end{tabular}

Note. $\mathrm{TAI}=$ traitement adaptatif de l'information

\section{Phase exploratoire de la TDD-EMDR}

La phase exploratoire suit des procédures TDD standard. Son objectif est le développement d'une compréhension des origines et de la nature de la réponse dépressive des patients. Comme la TDD n'est pas fondée sur le trauma, le recueil de l'histoire du patient se centre sur les principaux thèmes dépressogènes de la perte et de l'échec. Les phases EMDR standard 1 et 2 ne sont pas réalisées.

\section{Phase d'acceptation en TDD-EMDR}

Les phases EMDR 3 à 8 (F. Shapiro, 2001) sont utilisées dans la phase d'acceptation, avec des modifications déterminées par la théorie et les objectifs de la TDD. La cible principale identifiée pour l'EMDR est le sens corporel de perte et d'échec, et non des expériences ou des événements particuliers. Plutôt que l'approche standard en trois volets, passé-présentfutur, nous nous centrons uniquement sur le passé et sur le présent car le thème de difficultés futures pourrait réactiver l'état d'esprit qui tend à protester. Plutôt que de recourir aux cognitions négative $(\mathrm{CN})$ et positive (PC) standard de l'EMDR, le cadre cognitif est aligné sur l'axe protestation-acceptation. On ne tente aucunement de réduire l'échelle SUD (unités subjectives de perturbation) à zéro car le but de la phase EMDR consiste à favoriser l'acceptation et je ne connais aucune preuve qu'un état d'esprit d'acceptation soit affectivement neutre ou positif. La phase 5 de l'EMDR (installation cognitive) est modifiée à l'aide de questions rhétoriques destinées à favoriser le désengagement de la protestation et à susciter l'acceptation. Aucune CP n'est identifiée et des SBL servent plutôt à installer l'acceptation. Les phases EMDR 6, 7 et 8 sont réalisées en suivant les procédures EMDR standard, bien que la phase 8 (réévaluation) soit utilisée pour évaluer la stabilité de l'acceptation et de vérifier une récurrence possible de la protestation. La décision de retourner à la cible ou de passer à la phase suivante se fonde sur cette évaluation. On considère que la phase d'acceptation est achevée lorsque le patient cesse d'exprimer des sentiments de protestation.

\section{Phase d'activation comportementale en TDD-EMDR}

Dans le protocole intégratif, cette phase est conforme aux procédures TDD standard, tel que déjà décrit

(Krupnik, 2014). De plus amples informations expliquant le protocole intégratif sont fournies dans les paragraphes ci-après. 


\section{Méthode}

La raison de la description de deux cas est le bénéfice apporté par leur comparaison. En suivant cette logique, je décris les cas parallèlement plutôt que de manière séquentielle, afin de souligner leurs différences et leurs similitudes.

\section{Participants}

Stéphanie est une femme afro-américaine mariée de 23 ans et membre en service actif de l'armée des États-Unis. Après qu'elle ait décrit des symptômes dépressifs à son gestionnaire, Stéphanie a été orientée vers la clinique de santé mentale d'une base militaire. Lors de sa première visite, le nouveau-né de Stéphanie avait un mois et demi. Il s'agissait de son deuxième enfant, l'aîné étant âgé de 14 mois. Stéphanie s'occupait seule de ses enfants cas son mari se trouvait en déploiement. Quand elle travaillait, les enfants se trouvaient dans une crèche. Stéphanie était suivie par deux intervenants : l'un pour les médicaments et l'autre pour une psychothérapie. Les séances de thérapie étaient hebdomadaires, tandis que la gestion médicamenteuse se déroulait sur une base mensuelle.

Sheila est une femme hispanique mariée de 23 ans et un membre en service actif de l'armée des ÉtatsUnis. Elle s'est adressée à la clinique quand elle a commencé à éprouver des symptômes dépressifs, lorsque son enfant était âgé d'un mois. Son premier enfant avait alors deux ans. Sheila assistait également à des séances hebdomadaires de psychothérapie ainsi qu'à des entretiens mensuels pour la gestion de son traitement médicamenteux.

Les deux femmes étaient dans l'armée depuis quatre ans environ ; elles n'avaient pas d'expérience de combat ni de stress professionnel inhabituel. Stéphanie était stationnée loin de sa famille d'origine et entretenait surtout une relation à distance avec elle, tandis que Sheila avait tant sa propre famille que celle de son mari à proximité. Tous les enfants étaient globalement en bonne santé et aucune des mères ne souffrait de complications médicales importantes.

\section{Motif de la consultation}

Les tableaux cliniques partageaient de nombreux points communs. Les symptômes comprenaient des insomnies, une perte d'appétit, une concentration amoindrie, une anxiété réactionnelle accrue, une humeur dysthymique persistante, un sentiment d'inutilité, de la culpabilité, une perte d'intérêt et de motivation, un isolement social, une fatigue importante et une anhédonie. Les deux femmes niaient toute idéation suicidaire active et tout sentiment agressif envers leurs enfants.

Leurs présentations cliniques présentaient également des différences. Stéphanie se plaignait du sentiment d'être débordée par le fait d'occuper un poste à temps plein et d'élever seule ses deux enfants. Son état émotionnel était dominé par la tristesse, la solitude, la honte et l'épuisement. Sheila, par contre, se plaignait davantage de sa volatilité émotionnelle, avec une irritabilité chronique et des crises de larmes. Elle était troublée par des épisodes de colère envers ses enfants, se traduisant par des cris, même envers le nouveau-né. Même si son mari et sa famille l'aidait à s'occuper de ses enfants, elle était préoccupée par des difficultés conjugales. Si Stéphanie paraissait plus repliée sur elle-même et plus abattue, Sheila semblait davantage blessée, angoissée et énervée. En dehors du tempérament et de la culture, les circonstances et les antécédents personnels ont pu participer à créer ces différences.

\section{Histoire des patients}

Stéphanie est née dans le Midwest dans une famille intacte, la deuxième de trois enfants. Elle ne rapportait pas de violence, que ce soit dans l'enfance ou plus récemment. C'était une bonne élève avec des amis assez nombreux. Peu après avoir réussi ses études secondaires, Stéphanie s'est engagée dans l'armée, en quête d'indépendance vis-à-vis de sa famille. Après deux années de service actif, elle a épousé un militaire, et leur premier enfant est né environ six mois plus tard. Stéphanie a accouché de leur second enfant un an plus tard. Son mari a été déployé quand le bébé avait une semaine. Stéphanie a présenté des symptômes de la dépression peu après. Ils ont évolué au point de commencer à affecter sa performance au travail et la qualité des soins procurés aux enfants, et Stéphanie a dû demander de l'aide à la clinique de santé mentale. Elle n'avait pas d'antécédents de toxicomanie ou d'alcoolisme, ni d'antécédents familiaux de maladie mentale. Il s'agissait de sa première rencontre avec des professionnels de la santé mentale.

L'histoire de Sheila révélait une vie plus troublée. Elle a grandi dans une grande ville, la dernière de deux enfants, avec des parents qui n'ont jamais connu de vie familiale stable et dont la relation a comporté de nombreuses ruptures et réconciliations pendant toute son enfance. Elle a subi des agressions sexuelles répétées à l'âge de huit ans, de la part d'un garçon de six ans son aîné. Sheila se sentait négligée par ses parents lorsqu'elle était enfant mais était proche de sa grand-mère maternelle, la seule personne stable 
dans sa vie. La grand-mère est décédée quand Sheila avait 13 ans ; à partir de ce moment, Sheila a commencé à manifester des conduites sexualisées. Elle était souvent sanctionnée à l'école, étant considérée comme une " enfant en difficulté ", et ses rares amis lui faisaient subir des violences psychologiques. Sheila avait également le sentiment que sa mère, froide et distante, l'avait maltraitée émotionnellement. Elle a obtenu son diplôme d'études secondaires avec des notes médiocres. Elle s'est tout de suite engagée dans les forces armées pour "échapper » à sa famille. Deux ans plus tard, elle a épousé un militaire ; leur premier enfant est né peu de temps après, le second dix-huit mois plus tard. Sheila a commencé à éprouver une instabilité émotionnelle lors du troisième trimestre de sa grossesse, quand elle a appris que son mari l'avait trompée, et leur relation est devenue tendue. Ce n'est qu'à la naissance de l'enfant que ses symptômes ont progressé jusqu'à un tableau cliniquement significatif, ce qui l'a poussée à chercher un traitement. Sheila ne rapportait pas d'antécédents de toxicomanie ou d'alcoolisme, ni d'antécédents familiaux de problèmes de santé mentale. Malgré son enfance difficile, il s'agissait de sa première expérience avec des services de santé mentale. Son mari devait être déployé quatre mois après la naissance de leur second enfant.

\section{Évaluation}

Les diagnostics ont été établis par le psychothérapeute et confirmés par le psychiatre traitant, selon les critères du DSM-IV-TR (Diagnostic and Statistical Manual of Mental Disorders, $4^{e}$ édition, texte révisé ; APA, 2000) car le traitement a eu lieu avant la publication du DSM-5. Les deux femmes répondaient aux critères $\mathrm{A}$ à $\mathrm{E}$ d'un épisode dépressif majeur selon le DSM-IV. Elles rapportaient les symptômes 1, 2, 4, 6, 7 et 8 du critère $A$ avec une durée supérieure à un mois. Elles ne décrivaient aucun antécédent de sautes d'humeur importantes.

Les symptômes de Stéphanie sont apparus au cours du premier mois suivant la naissance de son deuxième enfant, dans un contexte de stress postpartum, comprenant le départ de son mari peu après l'arrivée du bébé et la nécessité de s'occuper de deux jeunes enfants en tant que parent isolé. Ces circonstances représentent un cas typique de la dépression post-partum, après la naissance d'un enfant dont la mère dispose de ressources limitées.

Le cas de Sheila correspond également à la notion de l'accouchement d'une mère dont les ressources sont compromises. Même si son mari était présent pendant et après la grossesse, son infidélité a fortement entamé la confiance qu'éprouvait Sheila en son avenir. Elle avait par moments envisagé le divorce. Son état mental s'est aussi détérioré peu après la naissance de son deuxième enfant.

La clinique fait habituellement un dépistage de l'ESPT chez tous les nouveaux patients, à l'aide des entretiens cliniques et d'une échelle d'évaluation de l'ESPT (PCL ; Weathers, Herman, Huska \& Keane, 1993). Les deux patientes ont obtenu un score inférieur au seuil significatif de 50 sur la PCL (Stephanie $=27$, Sheila $=42$ ) et aucune ne répondait aux critères d'un diagnostic d'ESPT. Même si Sheila rapportait des expériences traumatiques dans l'enfance, les agressions sexuelles n'avaient pas été violentes mais seulement forcées, et elle ne présentait pas de symptôme important spécifique au trauma, que ce soit avant ou pendant le traitement, à l'exception du critère $\mathrm{D}$ (altérations négatives de la cognition et de l'humeur) nouvellement ajouté (DSM-5). Elle reconnaissait le mal que ces agressions avaient causé au niveau de sa personnalité et de ses relations interpersonnelles. Au vu des présentations cliniques, les diagnostics multiaxiaux complets étaient

Stéphanie

Axe I - trouble dépressif majeur avec apparition post-partum

Axe II - pas de diagnostic

Axe III - pas de diagnostic

Axe IV - problème lié à une étape de vie

Axe $\mathrm{V}-61$

Sheila

Axe I - trouble dépressif majeur avec apparition post-partum

Axe II - pas de diagnostic

Axe III - pas de diagnostic

Axes IV - problème relationnel avec le conjoint

Axe $\mathrm{V}-65$

Outils d'évaluation. Le psychothérapeute a effectué à la fois la thérapie et les évaluations. Quatre outils psychométriques d'autoévaluation ont permis de suivre la dynamique des progrès des patientes à cinq moments déterminés : prétraitement, pendant le traitement, posttraitement, suivi après trois mois et suivi après six mois (figures 1 et 2). Les scores des figures sont présentés sous forme de pourcentage des scores prétraitement (considérés comme $100 \%$ ) afin de présenter simultanément la dynamique des différentes mesures. Les inventaires de Beck pour l'anxiété (Beck Anxiety Inventory [BAI]) et la dépression (Beck Depression Inventory-II [BDI-II]) ont permis de suivre les niveaux de symptômes anxieux et dépressifs, l'échelle "Échelle de satisfaction de vie » (Ladder of Life Satisfaction Scale) d'estimer la satisfaction 


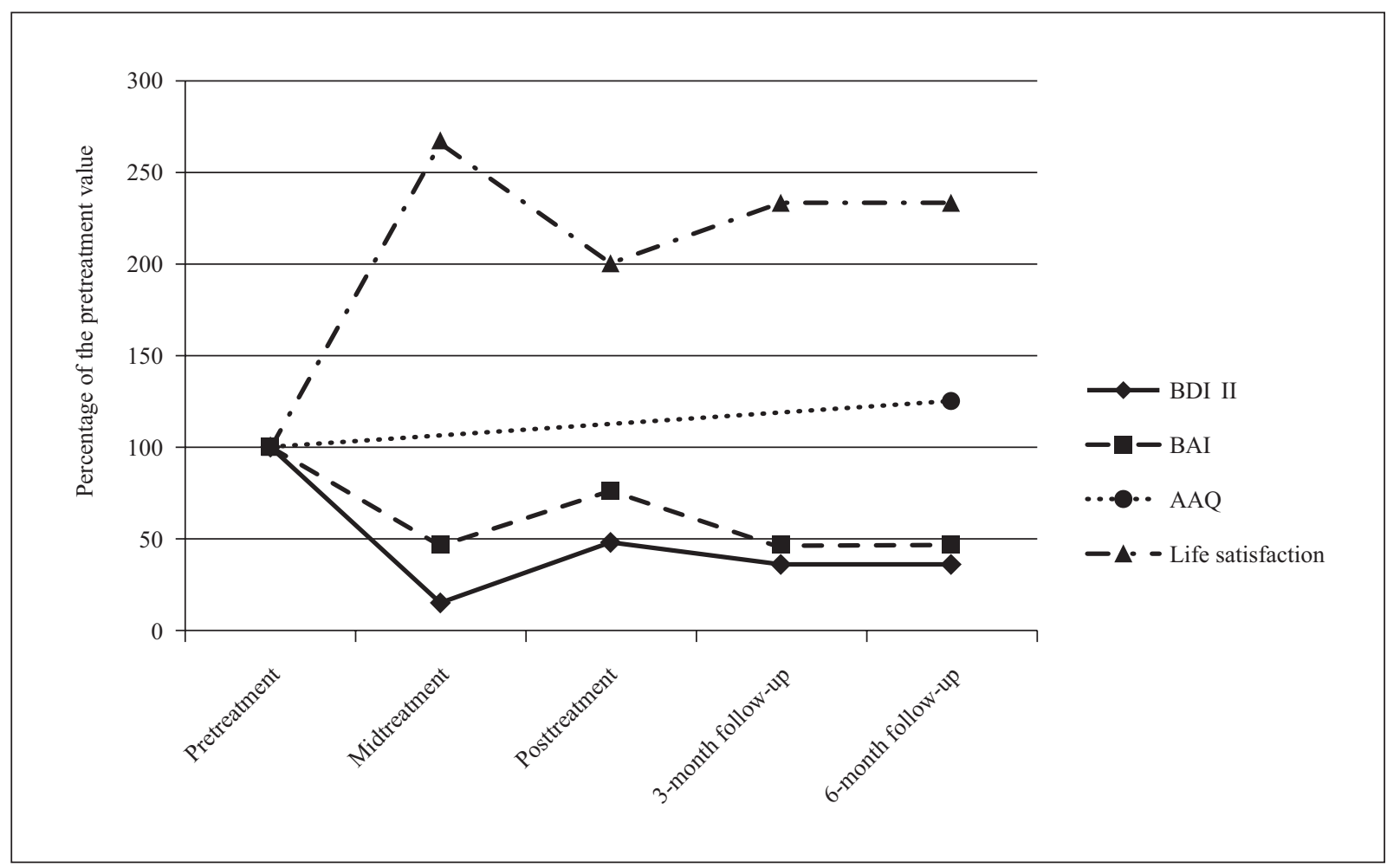

FIGURE 1. Stéphanie : l'évolution des notes d'autoévaluation exprimées sous forme de pourcentage des valeurs prétraitement. Note. Percentage of the pretreatment value $=$ pourcentage de la valeur prétraitement ; Pretreatment $=$ prétraitement ; Midtreatment $=$ mi-traitement $=$ séance $3 ;$ posttreatment $=$ post-traitement $=$ séance $10 ; 3$-month follow-up $=$ suivi après 3 mois ; 6-month follow-up = suivi après 6 mois ; BDI-II = Beck Depression Inventory-II $=$ inventaire de dépression de Beck ; $\mathrm{BAI}=$ Beck Anxiety Inventory $=$ inventaire d'anxiété de Beck $; \mathrm{AAQ}=$ Acceptance and Action Questionnaire $=$ questionnaire d'acceptation et d'action ; Life satisfaction = Ladder of Life Satisfaction Scale = échelle de satisfaction de vie de Cantril.

de vie générale, et le questionnaire d'acceptation et d'action (Acceptance and Action Questionnaire [AAQ]) d'évaluer les niveaux de disposition à l'acceptation.

Le BDI-II est un questionnaire à choix multiples consistant en 21 items représentant différents symptômes de dépression, dont la sévérité est évaluée entre 0 et 3. Il possède une fiabilité test-retest élevée de $r=$ 0,93 et une cohérence interne de $a=0,92$ (A. T. Beck $\&$ Steer, 1990). Les deux échelles sont cotées en additionnant les notes des items individuels, produisant ainsi une valeur de la sévérité générale des symptômes.

L'échelle de satisfaction de vie de Cantril (1965) est une échelle de type Likert en 11 points, demandant aux personnes interrogées d'évaluer leur vie actuelle (de la pire possible à la meilleure possible). Elle possède une fiabilité test-retest de $r=0,54$ (Atkinson, 1982) et un coefficient de validité conceptuelle de 0,59 (F. M. Andrews \& Crandall, 1976).

J'ai utilisé la version en 49 items de l'AAQ, un questionnaire d'autoévaluation conçu pour mesurer la disposition évitante. Sa fiabilité test-retest était estimée à $r=0,65$ et sa cohérence interne à $a=0,70$ (Hayes et coll., 2004).

\section{Déroulement du traitement}

Le traitement a respecté un cadre TDD-EMDR, suivant une séquence de trois phases : exploration, acceptation et activation comportementale. Dans la phase d'acceptation, des interventions EMDR modifiées ont permis de favoriser une disposition portée à l'acceptation (voir la description lors de la discussion antérieure). Nous avons effectué le traitement lors de séances hebdomadaires de 60 minutes.

Le nombre de séries de SBL (mouvements oculaires saccadiques) variait d'une séance à l'autre, allant de huit à vingt-cinq.

Stéphanie : phase exploratoire. Dans le cas de Stéphanie, la phase exploratoire n'a nécessité qu'une séance et demie. Elle a rapidement identifié un sentiment d'échec comme facteur contributif principal de sa dépression. "J'ai le sentiment d'avoir échoué à un moment donné. » En explorant plus loin ce sentiment d'échec, Stéphanie a défini l'échec comme le fait de ne pas avoir répondu à ses attentes à son propre égard et le sentiment d'avoir été incapable de relever les défis de la maternité aussi bien qu'elle pensait qu'elle aurait 


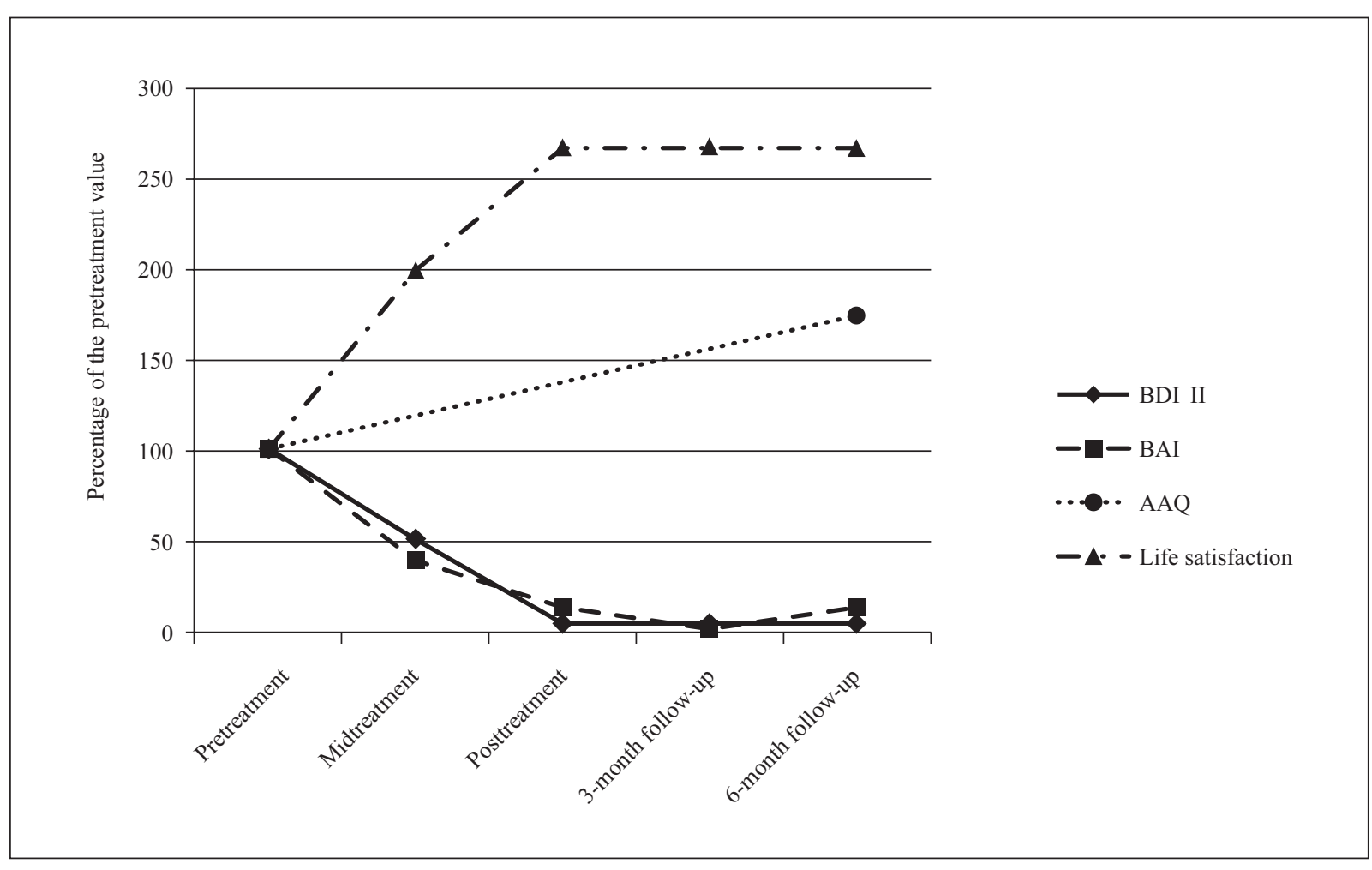

FIGURE 2. Sheila : l'évolution des notes d'autoévaluation exprimées sous forme de pourcentage des valeurs prétraitement. Note. Percentage of the pretreatment value $=$ pourcentage de la valeur prétraitement ; Pretreatment $=$ prétraitement ; Midtreatment $=$ mi-traitement $=$ séance $3 ;$ posttreatment $=$ post-traitement $=$ séance $10 ; 3$-month follow-up $=$ suivi après 3 mois ; 6-month follow-up = suivi après 6 mois ; BDI-II = Beck Depression Inventory-II = inventaire de dépression de Beck ; $\mathrm{BAI}=$ Beck Anxiety Inventory $=$ inventaire d'anxiété de Beck $; \mathrm{AAQ}=$ Acceptance and Action Questionnaire $=$ questionnaire d'acceptation et d'action ; Life satisfaction = Ladder of Life Satisfaction Scale = échelle de satisfaction de vie de Cantril

dû y parvenir. Son sentiment d'échec a conduit à des émotions de honte et de culpabilité. Ceci l'a amenée à remettre en question sa décision de se marier et de fonder une famille. Plus tard dans le traitement, elle a reconnu qu'elle se sentait inférieure à sa grande sœur qu'elle percevait comme avançant dans la vie, excellant dans sa carrière en reportant tout projet de fonder une famille. "Avant j'étais si déterminée et motivée mais ces derniers temps, je me suis centrée sur mon mari » disait-elle avec un air triste empli de regret. Stéphanie reconnaissait qu'elle s'est sentie échouer non seulement en raison des difficultés d'un parent isolé mais aussi au niveau de son objectif d'être une femme autonome qui réussit, comme sa sœur aînée. Plusieurs facteurs semblent avoir contribué à la facilité de ses prises de conscience, y compris une grande intelligence, une propension à l'introspection, une absence de résistance notable au changement et un faible niveau de défenses. Quand Stéphanie a commencé son traitement, son déni initial quant au sentiment d'être débordée et démunie s'était déjà dissipé. Par conséquent, elle avait déjà pris le premier pas vers l'acceptation de son échec. En même temps, sa protestation paraissait toujours active, tel que manifesté par des déclarations telles que " je veux que les choses reviennent à la normale ... je veux me reprendre en main mais je ne sais pas quoi faire ...»

Stéphanie : phase d'acceptation. Lors de la deuxième séance, nous avons ciblé en EMDR le sentiment d'échec de Stéphanie. La cible n'était pas une expérience ou un souvenir passé mais plutôt le sens corporel d'échec, y compris le composants cognitifs et émotionnels de ce sentiment. Stéphanie était invitée à se centrer sur l'idée de son échec et à identifier les émotions, les sensations et les cognitions ainsi suscitées. Elle a rapporté une émotion de tristesse, évaluée à 7 sur l'échelle SUD de 0 à 10 (où $0=$ aucune perturbation et $10=$ la pire perturbation possible) et la cognition était : « Je dois faire quelque chose ». Cette affirmation était considérée comme l'expression d'une protestation. Le traitement intégré se distingue de l'EMDR standard en ce qu'il ne demande pas aux patients de formuler une $\mathrm{CN}$ et une CP. Leurs cognitions sont plutôt considérées comme des manifestations de protestation ou d'acceptation ; tandis que 
les premières sont remises en question, les dernières sont encouragées. Stéphanie décrivait une sensation d'oppression thoracique comme accompagnant son affirmation.

Le traitement a démarré par une série d'environ 30 mouvements oculaires saccadiques et elle a rapporté que son esprit s'était vidé. On l'a invitée à revenir à la cible et les SBL ont été répétées, après quoi Stéphanie a rapporté la même pensée : "Je dois faire quelque chose ".

À ce moment, la psychothérapeute a demandé ce qu'elle pouvait faire, une série de SBL a suivi, et Stéphanie a prononcé une phrase d'acceptation : «Il n'y a rien que je puisse faire ". Elle a ensuite traversé une série de SBL en rapportant de manière consécutive : "Je ne peux rien faire d'autre que ce que je fais déjà ... Accepte-le et lâche prise ... maintenant je me sens calme ... Je m'inquiète de l'issue de ce que je vis actuellement ... c'est assez bête ... » Ces affirmations semblent refléter la dialectique intérieure entre la protestation et l'acceptation. La séance s'est terminée à ce moment-ci ; le SUD était descendu à 4 ; sa poitrine comportait toujours une sensation d'oppression.

À la séance suivante, Stéphanie rapportait une amélioration de son état avec une motivation accrue, une meilleure concentration et la capacité à apprécier certaines activités. Elle se plaignait toujours d'insomnie et d'anxiété. Quand elle était invitée à se centrer sur son sentiment dominant, Stéphanie a rapporté se sentir à la fois sans espoir et anxieuse (avec un SUD = 5) avec la pensée "je pourrais retomber en dépression », et une oppression thoracique associée. À nouveau, Stéphanie a traversé plusieurs séries de SBL en rapportant le fil de ses pensées. Cette fois, une dynamique différente est apparue. Le traitement semblait refléter la dialectique du désespoir et de l'espoir, Stéphanie rapportant des messages alternant entre la peur et l'espoir, progressant vers une plus grande assurance, « je me sens mieux quand je m'imagine jouer avec ma fille ... je pense au mot 'pire' pour une raison ou pour une autre ... je me sens heureuse et triste . . . je me sens nerveuse, j'ai peur et je suis enthousiaste ... ça passera ; j'y arriverai ... peur de l'échec, un sentiment de tristesse et d'échec ... . Je dois le vaincre, ne pas abandonner ... . je surmonterai tout ça, mais je dois y croire ... » À la fin de cette séance, le SUD de Stéphanie était descendu à 1 et l'oppression s'était dissipée. Cette fois, le psychothérapeute n'a pas cadré sa cognition mais a laissé le traitement se dérouler librement sans beaucoup intervenir, hormis pour renvoyer de temps en temps Stéphanie à ses propres affirmations d'assurance.

Lors de la séance suivante, Stéphanie a encore décrit une amélioration et a rempli une autre série d'outils d'autoévaluation (figure 1). Son score indiquait une nette amélioration, d'autant plus remarquable qu'il ne s'agissait que de la troisième séance.

Stéphanie: phased'activation comportementale. Les trois séances suivantes n'ont pas comporté d'intervention EMDR mais Stéphanie est passée à la phase d'activation comportementale, augmentant ses activités de loisirs dont une reprise de la lecture, des sorties dans un parc avec ses enfants, du sport et des échanges plus fréquents avec ses parents. Ses " tâches " comportementales à effectuer entre les séances étaient passées en revue et tout évitement était identifié et discuté. Elle s'appliquait également à développer une meilleure compréhension et à revoir son appréciation de sa situation pendant la partie non structurée des séances. Stéphanie continuait à rapporter un état amélioré par des phrases telles que " j'étais heureuse la plupart du temps ... j’apprécie le temps que je passe avec les enfants, je ne me sens plus submergée ". De manière peut-être plus importante, elle prononçait spontanément des affirmations d'acceptation comme " je n'ai jamais été aussi contente de là où j'en suis ... je suis bien comme je suis ». Stéphanie rapportait également une amélioration générale de son sommeil, de son humeur et de sa motivation ainsi que plus de plaisir.

Stéphanie : phase d'acceptation. À la septième séance, Stéphanie vivait une légère rechute des symptômes anxieux, de fatigue, d'une concentration réduite et d'agitation ; trois séances supplémentaires d'interventions EMDR ont suivi. À chaque séance, le sentiment dominant comprenant les cognitions et sensations somatiques associées était ciblé en vue de son retraitement, comme décrit auparavant. Les thèmes prévalents étaient les mêmes : un sentiment d'échec et de déception à son propre égard et l'impression d'être submergée par le stress. Les affirmations de Stéphanie reflétaient à nouveau son conflit intérieur entre le sentiment de désespoir et d'échec d'une part et des messages de confiance et d'assurance d'autre part : " toutes mes réalisations n'ont mené à rien . . . la vie ne se résume pas à ça . . . ma famille est fière de moi ... je devrais être fière de moi . . . je dois me centrer sur ce qui est important . . . je dois aller mieux pour moi et pour mes enfants ... " Il y avait aussi des expressions d'acceptation et d'optimisme, généralement vers la fin des séances, " je dois apprendre à lâcher prise ... parfois les choses qui se passent sont hors de mon contrôle ... tout va s'arranger . .. ». L'épisode de réapparition des symptômes de Stéphanie était reflété par ses scores aux échelles d'autoévaluation (figure 1). Stéphanie avait eu dix séances en tout (sans 
compter l'entretien initial) quand elle a senti qu'elle en avait retiré suffisamment de bénéfices et qu'elle a mis fin à la thérapie. À ce moment-là, elle rapportait une résolution de la plupart des symptômes et un fonctionnement satisfaisant. Ses scores aux outils d'autoévaluation indiquaient un bon progrès avec de légers symptômes résiduels de dépression et d'anxiété (figure 1), ce qui était attendu compte tenu du stress de devoir élever seule deux jeunes enfants tout en étant en service militaire actif.

Stéphanie : suivi. Les évaluations de suivi ont été menées par la psychothérapeute après trois et six mois. Stéphanie rapportait un état stable et ses scores aux échelles d'autoévaluation demeuraient stables (figure 1). Tout au long de sa psychothérapie, Stéphanie était suivie par un psychiatre pour la gestion médicamenteuse, ce qui constitue un facteur de confusion. Cependant son traitement par antidépresseurs n'était pas constant. Elle avait commencé à prendre de la sertraline mais avait cessé sa prise lors de la première séance de psychothérapie en raison d'effets secondaires gastro-intestinaux. Elle n'a pas repris de médicament avant la septième séance de thérapie, au moment où elle a connu la réapparition de ses symptômes et qu'elle avait peur de perdre les gains accomplis. Cette fois elle a pris du bupropion qu'elle tolérait bien. Elle a continué à voir le psychiatre occasionnellement pendant huit mois après la fin de sa psychothérapie, période au cours de laquelle son mari est rentré de son déploiement et que le couple s'est séparé. Il est intéressant de noter que si son psychiatre notait des plaintes de fatigue et de stress, Stéphanie n’a pas développé de second épisode dépressif mais a, au contraire, conservé une stabilité émotionnelle et un niveau de fonctionnement satisfaisant.

Sheila : phase exploratoire. La psychothérapie de Sheila s'est déroulée de manière similaire à celle de Stéphanie. Deux séances exploratoires ont été nécessaires avant de commencer l'EMDR. L'exploration a progressé depuis les stresseurs immédiats, tels que le sentiment d'être débordée par la responsabilité d'élever deux jeunes enfants tout en étant en service militaire actif ainsi que le ressentiment qui persistait envers son mari qui l'avait trompée pendant sa grossesse, vers des schémas dépressifs plus profonds et durables. Depuis qu'elle avait été agressée à l'âge de huit ans par un garçon de quatorze ans, Sheila luttait contre des sentiments de culpabilité et de honte. Elle avait toujours douté de sa valeur en tant que personne et en tant que femme. Elle reconnaissait qu'elle avait perdu le respect d'elle-même. Cette attitude a été renforcée au cours de son enfance par de multiples événements. Pendant son adolescence, ses parents la comparaient toujours défavorablement à sa sœur aînée, puis la grand-mère de Sheila, son principal soutien, est décédée, créant un sentiment d'abandon. La mère de Sheila, apparemment une personne narcissique, s'était toujours montrée froide et distante envers elle. De plus, Sheila se sentait marginalisée à l'école, fréquentant des filles «bizarres » et harcelantes, et adoptant des conduites sexualisées. Elle reconnaissait qu'elle avait eu plusieurs relations sexuelles avec des hommes qui avaient plusieurs partenaires. Sheila résumait sa situation ainsi : "J'ai toujours senti que j'étais une pute et une ratée. »

Sheila : phase d'acceptation. Lors de la troisième séance, Sheila a démarré l'EMDR en ciblant son sentiment d'échec en tant que personne et en tant que femme. Un thème qui a émergé spontanément, tandis qu'elle se centrait sur son échec, était la relation extraconjugale de son mari. Cette pensée suscitait une émotion de colère intense (SUD = 7). Au cours du retraitement, le thème d'infériorité à sa rivale est apparu : "Je la déteste ... je vois ses longs cheveux ondulants ... il voulait que je sois comme elle ... je ne serai jamais sûre de moi comme elle ... ils devraient être ensemble, plutôt que lui et moi ... je veux qu'elle soit aussi misérable que moi ... » D’un point de vue TDD, ceci indiquait une protestation, même si l'EMDR aurait pu l'approcher dans une perspective traumatique. La TDD et l'EMDR différent au niveau de leur conceptualisation de la psychopathologie (ce point est développé dans le paragraphe «Discussion » et dans le tableau 1.) À ce moment-là, le psychothérapeute a demandé à Sheila quelle était la raison pour laquelle son esprit se centrait sur ces pensées coléreuses, selon elle. Elle a dit qu'elle ne le savait pas mais le fil de sa pensée a pris un tournant. "Je ne devrais pas toujours penser à cette espèce de [juron] . . . ça n'aide pas mon estime de moi ... je dois trouver des façons de me sentir confiante et heureuse ... » Une séquence d'affirmations contradictoires a suivi : «Il me cache peut-être encore des choses ... je hais ce qu'il a fait mais je l'aime toujours ... je voudrais juste tout faire partir . .. » Ensuite quelques affirmations d'acceptation ont émergé : «Est-ce que je peux juste avancer ? J'aimerais tant tourner la page. » À la fin de la séance, le SUD de Sheila était descendu à 3.

Lors de la séance d'EMDR suivante, à la phase 8 , Sheila a décrit un rêve troublant dans lequel elle essayait en vain "d'attacher une corde épaisse à l'aide d'un fil fin ». Le rêve a été choisi comme cible et après la quatrième série de SBL, le thème de ses agressions sexuelles a émergé et elle a retraité ses émotions qui sont passées de la honte à la colère envers son 
agresseur. Le traitement ne s'est terminé que lors de la séance suivante, quand Sheila commençait à parvenir à une plus grande acceptation. «Je suis prête à tourner la page ... je dois me centrer sur ma formation ... » À ce moment-là, les scores des outils d'autoévaluation (figure 2) et ses propres déclarations indiquaient une amélioration importante de ses symptômes. Deux autres séances EMDR complètes (phases 3 à 8) ont suivi. Le retraitement de Sheila a révélé une lutte entre un sentiment de honte et le souhait de le dépasser, "Pourquoi je n'arriver pas à laisser tomber ... mon mari ne m'aurait pas épousée s'il avait connu mon passé ... j’ai l'impression de rompre avec moi-même . . . j'avais une ancre et maintenant j'ai l'impression de couper la corde qui me relie à cette ancre ... j'ai le sentiment de voler... »

Sheila : phase d'activation comportementale. Sheila a participé à six autres séances de TDD (pour un total de 12). Elle était encouragée à s'investir dans l'activation comportementale en élargissant la gamme de ses activités et de ses interactions sociales. Les séances étaient largement non structurées et incorporaient un développement supplémentaire de sa compréhension d'elle-même et de la thérapie de soutien.

Sheila : suivi. Les scores des échelles d'autoévaluation lors des évaluations de suivi montraient une amélioration stable (figure 2). Sheila recevait également un traitement médicamenteux pendant la psychothérapie. Son psychiatre lui a prescrit de la sertraline après sa première séance de thérapie et la posologie est demeurée stable tout au long de la psychothérapie et du suivi.

\section{Résultats}

Les cas décrits semblent avoir suivi une dynamique similaire, montrant une rémission relativement rapide (figures 1 et 2) avec une amélioration importante après trois (Stéphanie) ou quatre (Sheila) séances, avec une diminution de $75 \%$ et de $50 \%$ des scores du BDI-II, respectivement. En nombres absolus, les scores BDI-II ont diminué de sévères, Stéphanie $=33$, Sheila $=37$, à minimes, 12 et 1 respectivement, lors de la dernière évaluation de suivi. Cela constitue une baisse de plus de 20 unités, ce qui est considéré comme un changement conséquent (Hiroe et coll., 2005). De manière plus importante encore, dans les deux cas, j'ai observé une augmentation des scores d'acceptation (sur l'AAQ) entre le prétraitement et l'évaluation de suivi : $25 \%$ pour Stéphanie et $74 \%$ pour Sheila.

Bien qu'aucune comparaison directe ne puisse être réalisée à partir d'une étude de cas unique, en tant que repère, un essai sur la psychothérapie interpersonnelle dans le traitement de la dépression post-partum a montré une baisse de $25 \%$ des scores du BDI-II après quatre séances et de $54 \%$ post-traitement (O’Hara, Stuart, Gorman \& Wenzel, 2000). Il n'est pas clair quelle proportion de l'effet dans les cas décrits peut être attribuée au traitement par antidépresseurs parce que son efficacité a été démontrée pour la dépression post-partum (Appleby, Warner, Whitton $\&$ Faragher, 1997). Toutefois, Stéphanie n’a pas pris de médicament pendant les sept premières séances et son amélioration initiale ne pouvait pas être attribuée aux antidépresseurs.

Jusqu'à présent, j’ai utilisé la TDD-EMDR pour traiter six patients. En dehors des cas décrits ici, cela comprend deux cas de dépression majeure, un cas de trouble dépressif persistant et un de trouble dépressif non spécifié. La baisse pré-post moyenne des scores BDI-II était de $20(\sigma=10)$ et sa signification statistique a été confirmée par le test apparié de Wilcoxon $(w=0, p \leq 0,05)$.

\section{Discussion}

\section{Réconcilier les fondements théoriques}

La TDD-EMDR combine des traitements fondés sur des théories différentes. Les principales différences entre les approches théoriques sont résumées dans le tableau 1 et semblent complémentaires plutôt que contradictoires. L'EMDR a été développée en tant que thérapie centrée sur le trauma et se fonde sur la théorie de la psychopathologie et du changement thérapeutiques, le traitement adaptatif de l'information (F. Shapiro, 2001 ; Solomon \& Shapiro, 2008). La théorie TAI considère que la psychopathologie provient d'expériences traumatiques mal encodées et stockées de manière dysfonctionnelle. C'est pourquoi l'intervention thérapeutique vise à retraiter les souvenirs afin de les transformer et de les rendre mieux intégrés dans le réseau plus vaste de représentations d'expériences de vie.

La TDD a été spécialement conçue pour traiter la dépression selon une vision de la réponse dépressive comme une adaptation évoluée à une adversité insurmontable, lorsque l'organisme échoue à satisfaire ses besoins.

Les données préliminaires concordent avec l'attente d'une spécificité car la TDD n'était pas aussi efficace pour l'anxiété et pour des troubles de la personnalité qu'elle l'était pour la dépression (Krupnik, 2014).

Le rôle joué par les besoins inassouvis dans la psychopathologie fut reconnu il y a longtemps déjà (Maslow, 1943) et vient d'être soulignée à nouveau récemment (voir l’analyse de Flanagan, 2010). L’idée 
de la dépression comme une adaptation implique que la guérison fait partie de la réponse dépressive qui est donc conçue comme un processus biphasique dans lequel la phase de repli est suivie d'une phase de récupération. Mêmes des épisodes dépressifs non traités entrent en rémission dans les 12 mois en moyenne (p. ex. Furukawa, Kitamura \& Takahashi, 2000 ; Spijker et coll., 2002 ; Whiteford et coll., 2013). Ainsi le but de la TDD est de favoriser la réalisation complète des deux phases de la réponse dépressive. Une différence existe aussi au niveau de la notion d'adversité. Bien que l'EMDR soit utilisé avec succès pour traiter diverses affections qui ne relèvent pas de la catégorie des troubles liés au trauma selon le DSM-5, sa philosophie thérapeutique est tout de même centrée sur le trauma. Le trauma se définit comme "tout événement qui a un effet négatif durable sur le soi ou sur la psyché ", reliant ainsi presque toute pathologie affective à des « [traumas] petit ou grand T » (F. Shapiro \& Forrest, 2004, p. 14). La TDD ne se fonde pas sur le point de vue du trauma. Elle découle de la perspective de l'échec et de la perte en tant qu'adversité de vie " normale " plutôt que comme un trauma. La différence de conception de l'adversité n'est peut-être que philosophique mais elle oriente néanmoins l'approche psychothérapeutique.

La distinction entre les conceptions EMDR et TDD de la psychopathologie détermine la différence entre leurs visions du changement thérapeutique. Le mécanisme de changement selon le TAI est la transformation de souvenirs traumatiques au moyen d'un retraitement guidé. La TDD conçoit le désengagement de la protestation par un état d'esprit d'acceptation comme le principal mécanisme de changement thérapeutique.

\section{De la protestation à l'acceptation}

Dans la TDD, les troubles dépressifs sont compris comme une réponse dépressive interrompue. Ainsi, les interventions TDD ciblent les principaux points de transition du processus dépressif : la transition de la protestation à l'acceptation (phase d'acceptation) et de l'acceptation à une motivation restaurée (phase d'activation comportementale ; Krupnik, 2014). Dans la TDD-EMDR, les interventions EMDR sont employées dans la phase d'acceptation pour aider à la transition protestation-acceptation. À cette fin, le psychothérapeute guide le flux cognitif du matériel rapporté par les patients en suivant l'axe protestation-acceptation en posant des questions comme " Pensez-vous pouvoir y changer quelque chose? ", " Pouvez-vous y faire quelque chose ? » et « Pensez-vous avoir le contrôle là-dessus ? ».
Il peut sembler que dans les cas décrits, le thème de l'acceptation ait émergé spontanément au cours de la phase EMDR. En effet, il est apparu avec seulement des interventions minimes ; toutefois, l'apparente spontanéité peut être trompeuse. Le thème de l'acceptation était fortement présent, souvent de manière implicite, dans les deux premières phases de la TDDEMDR. Le psychothérapeute y faisait régulièrement référence pendant le développement d'une meilleure compréhension et dans le cadrage cognitif des interventions EMDR.

\section{(In)fidélité du traitement}

Dans la TDD-EMDR, nous nous écartons du protocole EMDR standard (F. Shapiro, 2001). Tel que déjà décrit, la nature des cibles, le cadre cognitif et le but visé pour l'évolution de l'affect du patient sont fondés sur la théorie TDD (tableau 1). Les interventions EMDR modifiées semblent avoir été efficaces dans les cas décrits, comme illustré par l'évolution des scores aux outils d'autoévaluation (figures 1 et 2 ) et par les affirmations verbales des patientes. De futures études de déconstruction pourraient élucider la contribution de chaque phase du traitement ainsi que la contribution de leur interaction. À tout le moins, une étude mesurant les résultats de chaque phase pourrait apporter des informations utiles à cet égard.

\section{Limites}

Une limite importante de cette étude provient de la nature préliminaire de ses résultats. Aucune conclusion concernant l'efficacité d'une psychothérapie ne peut être tirée d'une seule étude de cas. Pour cela, une étude contrôlée randomisée avec la participation de plusieurs psychothérapeutes serait nécessaire. Une limite déjà mentionnée était la pharmacothérapie simultanée au moyen d'antidépresseurs ; cependant, dans le cas de Stéphanie, la majeure partie de l'effet psychothérapeutique était atteinte avant sa prise de médicaments.

\section{Bibliographie}

American Psychiatric Association. (2000). Diagnostic and statistical manual of mental disorders ( $4^{\mathrm{e}}$ éd., texte révisé). Washington, DC: Auteur.

American Psychiatric Association. (2013). Diagnostic and statistical manual of mental disorders ( $5^{\mathrm{e}}$ éd.). Arlington, VA: Auteur.

Andrews, F. M. \& Crandall, R. (1976). The validity of measures of self-reported well-being. Social Indicators Research, 3, 1-19. 
Andrews, P. W. \& Thompson, J. A. (2009). The bright side of being blue: Depression as an adaptation for analyzing complex problems. Psychological Review, 116, 620-654.

Appleby, L., Warner, R., Whitton, A. \& Faragher, B. (1997). A controlled study of fluoxetine and cognitivebehavioural counselling in the treatment of postnatal depression. BMJ, 314, 932-936. http://dx.doi .org/10.1136/314.7085.932

Atkinson, T. (1982). The stability and validity of quality of life measures. Social Indicators Research, 10, 113-132.

Bae, H., Kim, D. \& Park, Y. C. (2008). Eye movement desensitization and reprocessing for adolescent depression. Psychiatry Investigation, 5, 60-65.

Beck, A. T. \& Steer, R. A. (1990). Manual for the Beck Anxiety Inventory. San Antonio, TX: The Psychological Corporation.

Beck, A. T., Steer, R. A. \& Brown, G. K. (1996). Manual for the Beck Depression Inventory-II. San Antonio, TX: The Psychological Corporation.

Beck, C. T. (2001). Predictors of postpartum depression: An update. Nursing Research, 50, 275-285.

Bisson, J., Roberts, N., Andrew, M., Cooper, R. \& Lewis, C. (2013). Psychological therapies for chronic posttraumatic stress disorder (PTSD) in adults. Cochrane Database of Systematic Reviews, (12), CD003388. http:/ / dx.doi.org/10.1002/14651858.CD003388.pub4

Bowlby, J. (1980). Attachment and loss, Vol. III: Loss: Sadness and depression. New York, NY: Basic Books.

Braun, S. R., Gregor, B. \& Tran, U. S. (2013). Comparing bona fide psychotherapies of depression in adults with two meta-analytical approaches. PloS One, 8, e68135. http: / / dx.doi.org/10.1371/journal.pone.0068135

Broad, R. D. \& Wheeler, K. (2006). An adult with childhood medical trauma treated with psychoanalytic psychotherapy and EMDR: A case study. Perspectives in Psychiatric Care, 42, 95-105.

Cantril, H. (1965). The pattern of human concerns. New Brunswick, NJ: Rutgers University Press.

Castonguay, L. G., Reid, J. J., Halperin, G. S. \& Goldfried, M. R. (2003). Psychotherapy integration. In G. Stricker \& T. A. Widiger (Eds.), Comprehensive handbook of psychology, Vol. 8: Clinical psychology (pp. 327-345). Hoboken, NJ: Wiley.

Cortés-Salim, P., González-Barrón, M. \& Romero-Gutiérrez, G. (2014). Psycho-emotional disorders in women with unplanned pregnancies. American Journal of Health Research, 2, 27-32.

Fava, M. (2003). Diagnosis and definition of treatmentresistant depression. Biological Psychiatry, 53, 649-659.

Feldman, R., Weller, A., Zagoory-Sharon, O. \& Levine, A. (2007). Evidence for a neuroendocrinological foundation of human affiliation: Plasma oxytocin levels across pregnancy and the postpartum period predict motherinfant bonding. Psychological Science, 18, 965-970. http: / / dx.doi.org/10.1111/j.1467-9280.2007.02010.x

Flanagan, C. M. (2010). The case for needs in psychotherapy. Journal of Psychotherapy Integration, 20, 1-36.
Furukawa, T. A., Kitamura, T., \& Takahashi, K. (2000). Time to recovery of an inception cohort with hitherto untreated unipolar major depressive episodes. The British Journal of Psychiatry, 177, 331-335.

Gaynes, B. N., Gavin, N., Meltzer-Brody, S., Lohr, K. N., Swinson, T., Gartlehner, G., . . . Miller, W. C. (2005). Perinatal depression: Prevalence, screening accuracy, and screening outcomes: Summary (AHRQ Publication No. 05-E006-2). Rockville, MD: Agency for Healthcare Research and Quality.

Gilbert, P. (2006). Evolution and depression: Issues and implications. Psychological Medicine, 36, 287-297.

Gilbert, P. \& Allan, S. (1998). The role of defeat and entrapment (arrested flight) in depression: An exploration of an evolutionary view. Psychological Medicine, 28, 585-598.

Giosan, C., Cobeanu, O., Mogoase, C., Muresan, V., Malta, L. S., Wyka, K. \& Szentagotai, A. (2014). Evolutionary cognitive therapy versus standard cognitive therapy for depression: A protocol for a blinded, randomized, superiority clinical trial. Trials, 15, 83. http://dx.doi .org/10.1186/1745-6215-15-83

Grey, E. (2011). A pilot study of concentrated EMDR: A brief report. Journal of EMDR Practice and Research, 5, 14-24. http: / / dx.doi.org/10.1891/1933-3196.5.1.14

Hagen, E. H. (1999). The functions of postpartum depression. Evolution and Human Behavior, 20, 325-359.

Harper, M. (2012). Taming the amygdala: An EEG analysis of exposure therapy for the traumatized. Traumatology: An International Journal, 18, 61-74. http://dx.doi .org/ 10.1177/1534765611429082

Hayes, S. C., Strosahl, K., Wilson, K. G., Bissett, R. T., Polusny, M. A., Dykstra, T. A., . . Bond, F. W. (2004). Measuring experiential avoidance: A preliminary test of a working model. The Psychological Record, 54, 553-578.

Hiroe, T., Kojima, M., Yamamoto, I., Nojima, S., Kinoshita, Y., Hashimoto, N., . . Furukawa, T. A. (2005). Gradations of clinical severity and sensitivity to change assessed with the Beck Depression Inventory-II in Japanese patients with depression. Psychiatry Research, 135, 229235. http: / / dx.doi.org/10.1016/j.psychres.2004.03.014

Hofmann, A., Hilgers, A., Lehnung, M., Liebermann, P., Ostacoli, L., Schneider, W. \& Hase, M. (2014). Eye movement desensitization and reprocessing as an adjunctive treatment of unipolar depression: A controlled study. Journal of EMDR Practice and Research, 8, 103-112. http: / / dx.doi.org/10.1891/1933-3196.8.3.103

Hofmann, S. G., Sawyer, A. T., Witt, A. A. \& Oh, D. (2010). The effect of mindfulness-based therapy on anxiety and depression: A meta-analytic review. Journal of Consulting and Clinical Psychology, 78, 169-183.

Holtforth, M. G., Hayes, A. M., Sutter, M., Wilm, K., Schmied, E., Laurenceau, J.-P. \& Caspar, F. (2012). Fostering cognitive-emotional processing in the treatment of depression: A preliminary investigation in exposure- based cognitive therapy. Psychotherapy and Psychosomatics, 81, 259-260. http://dx.doi .org/10.1159/000336813 
Jacobson, N. S., Martell, C. R. \& Dimidjian, S. (2001). Behavioral activation treatment for depression: Re- turning to contextual roots. Clinical Psychology: Science and Practice, 8, 255-270.

Jorm, A., Allen, N., Morgan, A. \& Purcell, R. (2009). A guide to what works for depression. Melbourne, Australia: Beyondblue.

Keller, M. C. \& Nesse, R. M. (2006). The evolutionary significance of depressive symptoms: Different adverse situations lead to different depressive symptom patterns. Journal of Personality and Social Psychology, 91, 316-330.

Krupnik, V. (2014). A novel therapeutic frame for treating depression in group treating depression downhill. SAGE Open, 4, 1-12. http://dx.doi.org/ $10.1177 / 2158244014523793$

Krupnik, V. (2015). Integrating EMDR into an evolutionary based therapy for depression: A case study. Clinical Case Reports, 3, 301-307. http: / dx.doi.org/10.1002/ ccr3.228

Kübler-Ross, E. (1997). On death and dying. New York, NY: Scribner Classics.

Maslow, A. H. (1943). A theory of human motivation. Psychological Review, 50, 370-396. http://dx.doi .org/10.1037/h0054346

Messer, S. B. (2001). Introduction to the special issue on assimilative integration. Journal of Psychotherapy Integration, 11, 1-4.

Murray, L., Arteche, A., Fearon, P., Halligan, S., Goodyer, I. \& Cooper, P. (2011). Maternal postnatal depression and the development of depression in offspring up to 16 years of age. Journal of the American Academy of Child \& Adolescent Psychiatry, 50, 460-470.

Nettle, D. (2004). Evolutionary origins of depression: A review and reformulation. Journal of Affective Disorders, 81, 91-102.

O’Hara, M. W., Stuart, S., Gorman, L. L., \& Wenzel, A. (2000). Efficacy of interpersonal psychotherapy for postpartum depression. Archives of General Psychiatry, 57, 1039-1045.

Rush, A. J., Trivedi, M. H., Wisniewski, S. R., Stewart, J. W., Nierenberg, A. A., Thase, M. E., . . Luther, J. F. (2006). Bupropion-SR, sertraline, or venlafaxine-XR after failure of SSRIs for depression. The New England Journal of Medicine, 354, 1231-1242.

Shapiro, F. (1989). Eye movement desensitization: A new treatment for post-traumatic stress disorder. Journal of Behavior Therapy and Experimental Psychiatry, 20, 211-217.

Shapiro, F. (2001). Eye movement desensitization and reprocessing (EMDR): Basic principles, protocols and procedures ( 2 éd.). New York, NY: Guilford Press.

Shapiro, F. (2014). The role of eye movement desensitization and reprocessing (EMDR) therapy in medicine: Addressing the psychological and physical symptoms stemming from adverse life experiences. The Permanente Journal, 18, 71-77. http: / dx.doi.org/10.7812 / TPP/13-098

Shapiro, F. \& Forrest, M. S. (2004). EMDR: The breakthrough therapy for overcoming anxiety, stress, and trauma. New York, NY: Basic Books.
Shapiro, R. E. (2009). EMDR solutions II: For depression, eating disorders, performance, and more. New York, NY: Norton.

Shedler, J. (2010). The efficacy of psychodynamic psychotherapy. American Psychologist, 65, 98-109.

Skrundz, M., Bolten, M., Nast, I., Hellhammer, D. H., \& Meinlschmidt, G. (2011). Plasma oxytocin concentration during pregnancy is associated with development of postpartum depression. Neuropsychopharmacology, 36, 1886-1893. http: / / dx.doi.org/10.1038/npp.2011.74

Solomon, R. M., \& Shapiro, F. (2008). EMDR and the adaptive information processing model: Potential mechanisms of change. Journal of EMDR Practice and Research, 2, 315-325. http: / / dx.doi.org/10.1891/1933-3196.2.4.315

Spijker, J., de Graaf, R., Bijl, R. V., Beekman, A. T., Ormel, J. $\&$ Nolen, W. A. (2002). Duration of major depressive episodes in the general population: Results from The Netherlands Mental Health Survey and Incidence Study (NEMESIS). The British Journal of Psychiatry, 181, 208-213.

Uribe, M. E. R., Ramírez, E. O. L. \& Mena, I. J. (2010). Effect of the EMDR psychotherapeutic approach on emotional cognitive processing in patients with depression. The Spanish Journal of Psychology, 13, 396-405.

Watt, D. F. \& Panksepp, J. (2009). Depression: An evolutionarily conserved mechanism to terminate separation distress? A review of aminergic, peptidergic, and neural network perspectives. Neuropsychoanalysis, 11, 7-109.

Weathers, F. W., Litz, B. T., Herman, D. S., Huska, J. A. \& Keane, T. M. (1993, October). The PTSD checklist (PCL): Reliability, validity, and diagnostic utility. Paper presented at the Annual Meeting of the International Society for Traumatic Stress Studies, San Antonio, TX.

Whiteford, H. A., Harris, M. G., McKeon, G., Baxter, A., Pennell, C., Barendregt, J. J. \& Wang, J. (2013). Estimating remission from untreated major depression: A systematic review and meta-analysis. Psychological Medicine, 43, 1569-1585.

Williams, J. M. G., Teasdale, J. D., Segal, Z. V. \& KabatZinn, J. (2007). The mindful way through depression: Freeing yourself from chronic unhappiness. New York, NY: Guilford Press.

Remerciements. L'auteur remercie l'éditrice Dr Maxfield ainsi que deux experts anonymes pour l'aide qu'ils ont aimablement apportée à l'amélioration de la première version de cet article. Les cas décrits dans l'article ont été présentés pour la première fois lors du congrès de l'Association internationale d'EMDR (EMDRIA) en 2014 à Denver au Colorado.

Merci d'adresser toute correspondance concernant cet article à Valery Krupnik, $\mathrm{PhD}$, Department of Mental Health, Naval Hospital Camp Pendleton, Mental Health Department, Naval Hospital Camp Pendleton, 200 Mercy Circle, Camp Pendleton, CA 92055, États-Unis. Courriel : valery.y.krupnik.ctr@mail.mil 